\title{
RNA Interference and Epigenetic Control of Heterochromatin Assembly in Fission Yeast
}

\author{
H. CAM AND S.I.S. GREWAL \\ Laboratory of Molecular Cell Biology, National Cancer Institute, National Institutes of Health, \\ Bethesda, Maryland 20892
}

Large tracts of eukaryotic chromosomes contain no or relatively few genes and are often replete with repetitive elements. Many of these regions are heterochromatic in nature, being highly condensed and inaccessible to a variety of DNA-modifying factors, and therefore are generally thought to be synonymous with gene silencing. Importantly, a phenomenon of position effect variegation (PEV) first observed in flies, in which a euchromatic gene when juxtaposed to heterochromatin becomes repressed in a stochastic fashion but once silenced can be clonally inherited, suggests that cellular factors intimately associated with heterochromatin can stably maintain epigenetic gene silencing through multiple cell divisions without altering the underlying genetic sequence (Grewal and Elgin 2002).

PEV has also been observed in the fission yeast Schizosaccharomyces pombe whose genome contains large stretches of heterochromatin with properties similar to heterochromatin present in higher eukaryotes (Grewal 2000; Hall and Grewal 2003). Because of its rather compact genome and tractable genetic system, heterochromatin-mediated gene silencing is arguably best understood in this organism. In the last few years, great strides have been made in uncovering the molecular constituents of heterochromatin and how they effect epigenetic gene silencing. In particular, recent works from our lab and others have elucidated a number of molecules involved in heterochromatin formation, with the surprising discovery of the critical role of the RNA interference (RNAi) pathway in the targeting and assembly of heterochromatin at specific loci of the $S$. pombe genome. A basic picture of how heterochromatin assembles, propagates, and is stably maintained through mitosis and meiosis has begun to emerge.

\section{HETEROCHROMATIC REGIONS IN S. POMBE}

The three chromosomes of fission yeast contain several silenced domains that are constitutively associated with heterochromatin, namely the telomeres, centromeres, the mating-type ( $m a t)$ region, and the ribosomal DNA (rDNA) tandem loci (Grewal 2000; Thon and VerheinHansen 2000). Unlike the small centromeres of the budding yeast Saccharomyces cerevisiae, which can comprise only a few hundreds base pairs (bp) of DNA, centromeres in $S$. pombe are relatively large complex structures (35-110 kilobases [kb]) that resemble higher eukaryotic centromeres in structure and organization, having partitions of large inverted-repeat elements flanking the central core (cnt), a site of kinetochore assembly and microtubule attachment (Fig. 1A). Clusters of tRNA genes pepper within the inner (imr) domains, while tandem but variable copies of the $d g$ and $d h$ repeats, whose sequences are remarkably conserved among themselves, orient in an inverted symmetry within the centromeric outer (otr) domains surrounding both sides of the central core of each chromosome (Takahashi et al. 1992). Reporter genes ectopically placed at the otr, imr, and cnt domains are transcriptionally silenced (Allshire et al. 1994).

Although less is known about the nature of telomeres and rDNA loci, evidence of PEV, suppression of DNA recombination, and common heterochromatin-associated factors localized to these regions as to the centromeres indicate similar mechanisms of heterochromatin formation operating at these loci as well. In contrast, the mating-type region of fission yeast located on the right side of the centromere of chromosome II is a well-studied locus containing several cis-acting elements that are known to contribute to transcriptional silencing at the mat locus (Fig. 1B). Heterochromatin spanning across the $K$ region, a 20 $\mathrm{kb}$ interval between mat 2 and mat 3 loci of the mating-type region, has been shown to be important for maintaining gene silencing and suppression of interchromosomal recombination. Interestingly, heterochromatin at the mating-type region has been implicated in the control of directionality of mating-type switching, in which matingtype information present at the upstream mat 1 locus is unidirectionally replaced with the opposite mating-type allele copied from donor cassettes present downstream at mat2 and mat 3 loci. In fact, genetic screens looking for matingtype switching mutants identified factors that also participate in heterochromatin assembly (Egel et al. 1984; Grewal and Klar 1997).

\section{HETEROCHROMATIN ASSEMBLY FACTORS IN FISSION YEAST}

Several trans-acting factors involved in effecting gene silencing at heterochromatic loci within the $S$. pombe genome have been identified (Table 1). These factors directly or indirectly affect heterochromatin assembly at these loci. Importantly, many of these factors have been shown to have homologs in higher eukaryotes that par- 
A
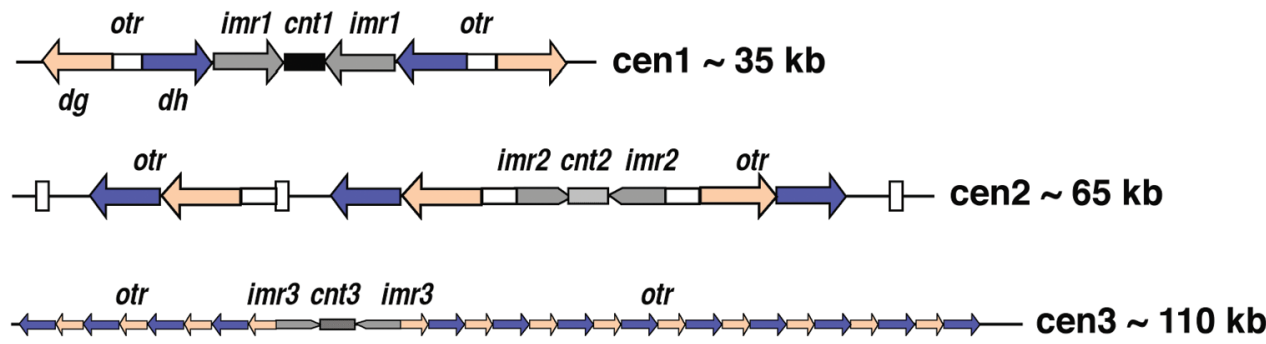

B

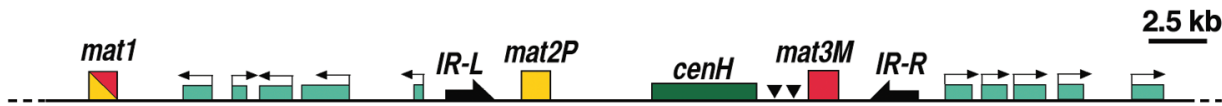

Figure 1. Diagrammatic representation of the $S$. pombe centromeres and the mating-type region. $(A)$ The central domain $(c n t)$ at each centromere is flanked by a series of inner ( $\mathrm{imr}$ ) and outer (otr) repeat elements. The otr regions contain $d g$ and $d h$ repeats that are highly conserved among themselves and double-stranded RNA (dsRNA) transcripts have been detected from these regions. $(B)$ The mating-type region contains three loci involved in mating-type switching-mat1, mat2, and mat3 - and a number of silent cis-acting elements, among the most dominant is $c e n H$, which lies within the $20-\mathrm{kb}$ mat2/3 interval, a heterochromatic region exhibiting both suppression of transcription and recombination. cen $H$ possesses $d g$ and $d h$ repeats that are highly homologous to centromeric $d g$ and $d h$ repeats. dsRNA transcripts whose regulation is RNAi dependent have also been detected at cenH. The double black triangles between cenH and mat3 indicate Atf1/Pcr1-binding sites.

take in heterochromatin formation as well. spSir2, Clr3, and $\mathrm{Clr} 6$ are histone deacetylases (HDACs), enzymes that remove acetyl group from histones' tails (Grewal et al. 1998; Bjerling et al. 2002; Nakayama et al. 2003; Shankaranarayana et al. 2003). spSir2 belongs to a special class of HDACs, present also in budding yeast and higher eukaryotes, that requires the cofactor nicotinamide adenine (NAD) for its catalytic activity (Shankaranarayana et al. 2003). Clr3 and Clr6 are homologous to mammalian HDACs class 1 (HDAC1 and HDAC2) and class 2 (HDAC2 and HDAC5), respectively. HDAC activity has been shown in many systems, including $S$. cerevisiae, to be a prerequisite for establishing a repressive chromatin state, and genome-wide analyses have indicated that heterochromatic regions tend to exhibit overall hypoacetylation relative to euchromatic regions (Kurdistani and Grunstein 2003; Schubeler et al. 2004).

Clr4 is homologous to Su(var)3-9 in Drosophila and SUV39H1 in mammals. Both loss of Clr4 and Su(var)39 suppress PEV in S. pombe and Drosophila, respectively
(Grewal and Elgin 2002). These proteins possess a conserved amino-terminal chromodomain and a carboxy-terminal SET domain. Clr4 has been shown to preferentially methylate lysine 9 residue on the amino tail of histone $\mathrm{H} 3$ (H3-K9) (Rea et al. 2000; Nakayama et al. 2001b). While the SET domain confers histone methyltransferase (HMT) activity on Clr4, the chromodomain is thought to be important for the localization of $\mathrm{Clr} 4$ to silenced loci (Nakayama et al. 2001b). Swi6 functions as a chromatin modifier and is structurally similar to heterochromatin protein 1 (HP1) of Drosophila and mammals (Lorentz et al. 1994). Swi6/HP1 contains a chromodomain at its amino terminus that has been shown to bind to H3-K9methyl residues created by Clr4/Suv39H1, a variable hinge region, and the chromoshadow domain at its carboxyl terminus. The chromoshadow domain of Swi6/HP1 promotes Swi6/HP1 oligomerization, which is thought to be important for chromatin compaction and heterochromatin spreading (Brasher et al. 2000; Cowieson et al. 2000; Wang et al. 2000). Chp1 and Chp2

Table 1. Factors Involved in Heterochromatin-mediated Silencing

\begin{tabular}{lll}
\hline Locus & Function & Motifs/similarity \\
\hline spSir2 & NAD-dependent histone deacetylase & S. cerevisiae, Drosophila, human Sir2 \\
clr1 & Putative DNA-binding protein & Three zinc fingers \\
clr2 & Unknown & No similarities in database \\
clr3 & Histone deacetylase & Human HDAC4 and HDAC5, . cerevisiae Hda1 \\
clr4 & Histone methyltransferase & SET domains and chromodomains/Drosophila Su(var)3-9 and \\
& & Polycomb, human SUV39H1 \\
clr6 & Histone deacetylase & Human HDAC1 and HDAC2, S. cerevisiae Rpd3 \\
swi6 & Chromatin modifier & Chromodomains and shadow domains/Drosophila and human \\
& & HP-1 and Polycomb \\
chp1 & Chromatin modifier & Chromodomain, homologous to Swi6 \\
chp2 & Chromatin modifier & Chromodomains and shadow domains, homologous to Swi6 \\
rik1 & Putative RNA/DNA-binding protein & 11 WD40-like repeats \\
\hline
\end{tabular}


are also chromodomain proteins. Chp2 is similar to Swi6 in structure possessing a chromodomain as well as a chromoshadow domain. Loss of Chp1, Chp2, or Swi6 results in derepression at a number of heterochromatic regions including at the centromeres and the rDNA tandem repeat loci (Thon and Verhein-Hansen 2000). Rik1, which shares homology to Ddb1, a group of evolutionarily conserved proteins involved in cullin-mediated ubiquitination and nucleotide excision repair (Tuzon et al. 2004), has been shown to be essential for Clr4 methylation of H3-K9 at heterochromatic loci (Nakayama et al. 2001b).

\section{HISTONE CODE AND HETEROCHROMATIN ASSEMBLY}

The basic unit of eukaryotic chromatin is nucleosome, an octamer of histone proteins made up of two molecules each of H2A, H2B, H3, and H4. A stretch of 146 bp of genomic DNA coils tightly around the carboxy-terminal two-third of the core histones, leaving the amino-terminal tails of histones to freely interact with other chromosomal factors. Certain key amino acid residues on the histone tails are targeted by a number of enzymes to a variety of posttranslational modifications such as acetylation, methylation, phosphorylation, ADP-ribosylation, and ubiquitylation. These modified residues have been shown to serve as markers that can recruit chromatin modifier proteins. Accumulating evidence suggests these covalent modifications on histone tails together may constitute a "histone code" that is read by nonhistone chromosomal proteins to effect changes ranging from a few nucleosomes at a locus to large-scale global organization of an entire chromosome (Strahl and Allis 2000; Jenuwein and Allis 2001). Recent findings in fission yeast and higher eukaryotes have led to a model of heterochromatin assembly based on a sequential series of histone modifications involving concerted hypoacetylation by HDACs and methylation by Clr4/Suv39H1 and other HMT proteins.

An important advance in which a molecular marker could be identified with heterochromatin occurred when work performed in our lab by Jun-ichi Nakayama, in collaboration with David Allis's group, provided the first in vivo evidence of $\mathrm{H} 3-\mathrm{K} 9$ methylation associated with heterochromatin (Nakayama et al. 2001b). Nakayama et al. detected H3-K9 methylation at the fission yeast's centromeres and the mating-type region, and this methylation pattern depends on the presence of Clr4: Loss of Clr4 completely abolishes H3-K9 methylation at these loci (Nakayama et al. 2001b). Moreover, point mutations in the SET domain as well as the carboxy-terminal postSET motif severely hinder the ability of Clr4 to methylate H3-K9 (Nakayama et al. 2001b). The discovery of stable methylation marks on heterochromatin-associated histone $\mathrm{H} 3-\mathrm{K} 9$ reveals that this may be part of the histone code, signaling to recruit heterochromatin assembly factors. Indeed, one protein that is capable of binding to methyl H3-K9 marks is Swi6/HP1. Swi6/HP1 can bind to methylated $\mathrm{H} 3-\mathrm{K} 9$ in vitro, but with preference for dimethyl and trimethyl $\mathrm{H} 3-\mathrm{K} 9$, which is thought to correspond to the degree of gene silencing (Bannister et al.
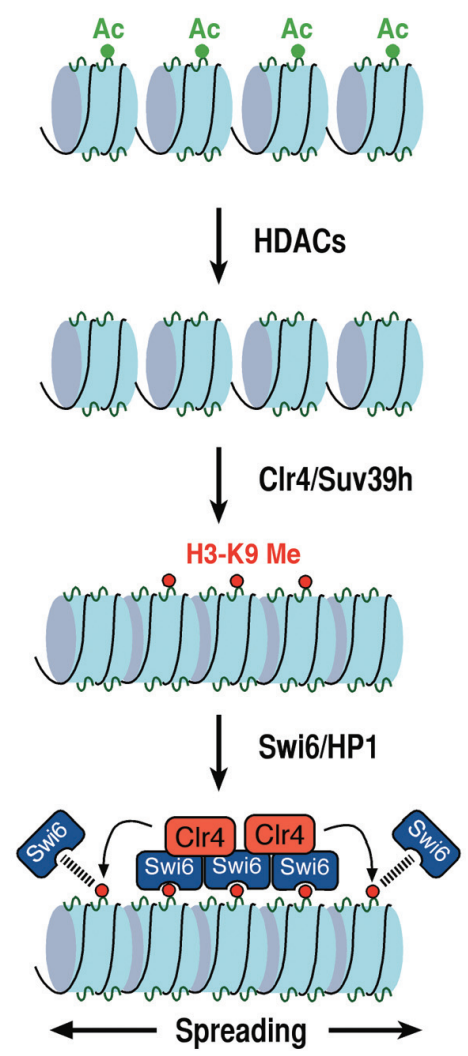

Figure 2. Stepwise model of heterochromatin assembly. Heterochromatin assembly is thought to require concerted deacetylation and methylation on the histones' tails. Deacetylation by the HDACs' enzymes creates a repressive chromatin state and allows histone methyltransferases such as $\mathrm{Clr} 4 / \mathrm{Suv} 39 \mathrm{~h} 1$ to methylate lysine 9 on histone H3 (H3-K9 Me). Methylation of H3-K9 provides the binding sites for Swi6/HP1 proteins, which, once bound to chromatin, can facilitate heterochromatin spreading to adjacent sequences.

2001; Lachner et al. 2001; Jacobs and Khorasanizadeh 2002; Nielsen et al. 2002). In fission yeast, localization of Swi6 to centromeric and the mating-type loci is entirely dependent on the presence of $\mathrm{H} 3-\mathrm{K} 9$ methylation (Nakayama et al. 2001b). Deletion of Clr4 or loss of Clr4 HMT activity delocalizes Swi6 from heterchromatic loci suggesting that the binding of Swi6 to chromatin is primarily dependent on Clr4 to methylate $\mathrm{H} 3-\mathrm{K} 9$ (Nakayama et al. 2001b). Interestingly, loss of Swi6 prevents the spreading of $\mathrm{H} 3-\mathrm{K} 9$ methylation throughout the mat locus while mutations in Clr4 attenuate Swi6 association with heterochromatin (Nakayama et al. 2001b; Hall et al. 2002). Together, these data support a model of heterochromatin assembly in which Swi6 initially localizes to chromatin via its binding to methylated H3-K9 established by $\mathrm{Clr} 4$ (Fig. 2). Once bound to methylated H3-K9, Swi6 can stimulate self-oligomerization through its chromoshadow domain and the recruitment of additional $\mathrm{Clr} 4$ proteins to methylate H3-K9 residues on adjacent nucleosomes (G. Xiao and S.I.S. Grewal, unpubl.), thereby creating more binding sites for Swi6. The result is a feedback loop that allows heterochromatin to spread to neighboring sequences (Fig. 2). 
Although methylation of H3-K9 by $\mathrm{Clr} 4$ seems to be the critical step in the assembly of heterochromatin, it is likely to be preceded by a series of deacetylation steps, by Clr3, spSir2, and Clr6 HDACs, because loss of any of these proteins either reduces or abolishes H3-K9 methylation altogether (Nakayama et al. 2001b, 2003; Shankaranarayana et al. 2003).

\section{RNAi-MEDIATED HETEROCHROMATIN FORMATION}

Repetitive DNA elements have long been known to play an important role in inducing heterochromatin (Selker 1999; Hsieh and Fire 2000; Hall and Grewal 2003). In fission yeast, a cenH element homologous to centromeric $d g$ and $d h$ repeats present within the mat $2 / 3$ interval contributes to epigenetic silencing of the matingtype region (Grewal and Klar 1996). Mutant strain carrying a deletion of $c e n H$ fails to efficiently establish heterochromatin at the mat locus (Grewal and Klar 1996, 1997). Insertion of cen $H$ confers repression on a reporter gene and induces heterochromatin at an ectopic euchromatic site (Ayoub et al. 2000). Recent findings suggest cenH and similar repetitive DNA elements collaborate with the RNAi machinery to induce heterochromatin formation at loci containing these repetitive elements (Hall et al. 2002; Hall and Grewal 2003).

The fission yeast contains three evolutionarily conserved components of the RNAi machinery-namely, Agol (a PAZ/Piwi family Argonaute), Dcr1 (a RNaseIIIlike enzyme Dicer), and Rdp1 (an RNA-dependent RNA polymerase) - and all have been found to be necessary for heterochromatin formation in $S$. pombe (Hall et al. 2002; Volpe et al. 2002). In RNAi mutants lacking either Ago1, Dcr1, or Rdp1, there is a complete loss of H3-K9 methylation and Swi6 localization at centromeric repeats and at cenH ectopic site (Hall et al. 2002; Volpe et al. 2002). Interestingly, H3-K9 methylation and Swi6 localization are apparently normal at the mating-type region in these RNAi mutants (Hall et al. 2002). However, in contrast to wild-type strains, RNAi mutants grown in the presence of a deacetylase inhibitor trichostatin A (TSA), which has been shown to remove epigenetic imprint of transcriptional silencing at the mat locus, fail to efficiently reestablish heterochromatin upon the removal of TSA (Hall et al. 2002). These results distinguish heterochromatin formation into two distinct phases: the initial targeting and nucleation of heterochromatin, and the spreading and maintenance of heterochromatin. Importantly, the results support the critical role for RNAi in the initial phase of heterochromatin formation, while at the mating-type region RNAi becomes dispensable at the subsequent phase of heterochromatin maintenance.

A multiprotein RNAi effector complex called RISC (RNA-induced silencing complex) has been biochemically characterized in Drosophila (Hammond et al. 2001; Murchison and Hannon 2004). RISC contains Argonaute and small interfering RNA (siRNA) molecules of 21-24 nucleotides (nt) cleaved by the enzyme Dicer from longer double-stranded RNA (dsRNA) transcripts (Bernstein et al. 2001). These siRNAs are thought to provide the specificity for RISC-targeted degradation or translational inhibition of mRNA and transcriptional repression. In fission yeast, siRNAs corresponding to centromeric $d g$ and $d h$ repeats have also been detected in wild-type cells (Reinhart and Bartel 2002). Interestingly, dsRNA transcripts derived from centromeric otr repeats and from cenH of the mating-type region, while not present in wild-type strains, could be detected in RNAi mutants, suggesting that in wild-type cells, dsRNA transcripts are actively being processed into siRNAs (Volpe et al. 2002; K. Noma and S. Grewal, unpubl.). However, the connection between siRNAs and heterochromatin formation was not readily understood until the recent purification of RITS (RNA-induced initiation of transcriptional gene silencing) in fission yeast (Verdel et al. 2004).

RITS is an RNAi effector complex containing Chp1, a heterochromatin-associated chromodomain protein that is required for H3-K9 methylation and Swi6 localization at the centromeres (Partridge et al. 2002), a novel protein Tas3, and importantly Agol and siRNAs (Verdel et al. 2004). RITS can localize to centromeric otr repeats in an RNAi-dependent manner. In the absence of Dcr1, RITS can still assemble but without siRNAs and can no longer localize to the centromeres, suggesting RITS-associated siRNAs might provide the locus-specific sequence that can guide RITS to designated genomic site. Loss of any of the RITS components abolishes H3-K9 methylation and Swi6 localization at the centromeres. These results

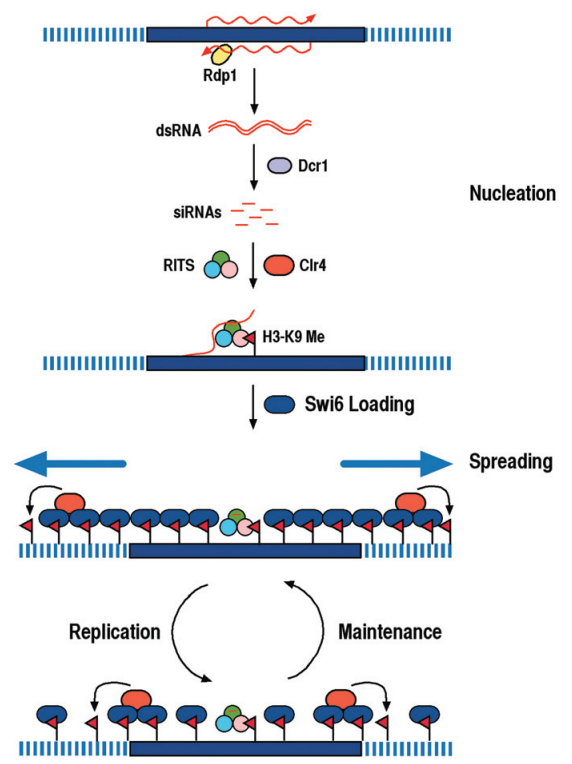

Figure 3. Model of RNAi-mediated heterochromatin formation in $S$. pombe. Double-stranded RNAs (dsRNAs) produced (possibly aided by Rdp1) from regions containing repetitive elements are recognized and converted into siRNAs by Dcr1. siRNAs contain the locus-specific sequence and, in complex with RITS or yet unknown protein(s), may facilitate the recruitment of heterochromatin assembly factors such as $\mathrm{Clr} 4$ to site with homologous sequence. Once H3-K9 has been methylated by Clr4, Swi6 can localize to the target site and stimulate the spreading and establishment of heterochromatin. 
implicate RITS as the complex that links the RNAi pathway to heterochromatin assembly, providing a cellular mechanism for how RNAi can induce heterochromatin formation at specific genomic loci. A basic picture of how the RNAi machinery can cooperate with chromatin modifier factors to assemble heterochromatin at regions producing dsRNAs can now be delineated as follows (Fig. 3).

The first step in RNAi-mediated heterochromatin assembly involves the synthesis of double-stranded transcripts from genomic regions containing highly repetitive elements. These transcripts are recognized and converted by Dcr1 into siRNAs. siRNAs in association with RITS facilitate in guiding RITS to genomic loci with homologous sequences. Once bound to chromatin, RITS can mediate the recruitment of histone modifier proteins including Clr4. Alternatively, RITS presumably recruits the RNAi machinery to the target site to promote the generation of siRNAs that in turn stimulates the recruitment of heterochromatin assembly factors. However, once $\mathrm{Clr} 4$ is recruited and the initial methylation of H3-K9 is established, Swi6 can abet in the spreading of heterochromatin in cis into neighboring sequences. The persistent presence of RITS on chromatin once heterochromatin has already been assembled suggests a posttranscriptional role for RITS (K. Noma and S. Grewal, unpubl.). Perhaps RITS participates in posttranscriptional repression and, conceivably in collaboration with Rdp1 and Dcr1, processes dsRNA into siRNAs, similar to the function of RISC seen in other systems (Murchison and Hannon 2004).

\section{RNAi-INDEPENDENT HETEROCHROMATIN FORMATION AT THE MATING-TYPE REGION}

While RNAi mutants display a complete loss of heterochromatin at the centromeres, heterochromatin and gene silencing are not affected at the mating-type region (Hall et al. 2002). This rather paradoxical result suggests that other cis-acting silent elements besides cenH could compensate for the loss of RNAi to maintain heterochromatin at the mat locus. Indeed, since RNAi and cenH act in the same pathway and cenH-deleted mutant strains containing a reporter gene inserted at the mat $2 / 3$ interval could still acquire an epigenetic off state, these observations suggest the existence of RNAi-independent factors that can initiate heterochromatin formation at the matingtype region in the absence of RNAi (Grewal and Klar 1997; Hall et al. 2002). Songtao Jia from our lab analyzed the mat2/3 interval and identified two heptamer binding sites for the transcription factor Atf1/Pcr1 located near the mat3 locus (Jia et al. 2004). Whereas RNAi mutants retain normal epigenetic silencing at the mating-type region, RNAi mutants lacking either Atf1 or Pcr1 exhibit a dramatic reduction in silencing, accompanied by significant decreases in H3-K9 methylation and Swi6 level at the mat locus (Jia et al. 2004). Chromatin immunoprecipitation (ChIP) assay confirms Atf1 and Pcr1 localization to mat3 region containing the Atf1/Pcr1-binding sites. Deletion of those sites negates the binding of Atf1 and Pcr1 to mat3 locus. Interestingly, Atf1/Pcr1 localization to the mating-type region is not affected in the absence of Swi6. Taken together, these results suggest that Atf1/Pcr1 can bind directly to the mat 3 region, and it acts probably upstream of Swi6 and is capable of recruiting heterochromatin assembly factors such as HDACs and Clr4 to nucleate heterochromatin formation independent of the RNAi pathway (Jia et al. 2004).

\section{PROPAGATION AND INHERITANCE OF HETEROCHROMATIC STRUCTURES}

Results from our studies of the fission yeast have revealed multiple parallel pathways that could lead to heterochromatin formation. Once heterochromatin is established, its propagation and stability need to be maintained with fidelity through multiple cell divisions without being "diluted" by macrocellular processes such as DNA replication and chromosome condensation and segregation. For example, during DNA replication, epigenetic imprint from one DNA molecule has to be transferred to the newly synthesized molecule. Since heterochromatin propagates in cis (Nakayama et al. 2000), this probably requires heterochromatin assembly factors to collaborate with the DNA replication machinery to reestablish epigenetic marks (imprinting) on the new DNA molecule as it is being synthesized. Alternatively, components of the DNA replication machinery such as proliferating cell nuclear antigen (PCNA) and chromatin assembly factor-1 (CAF1) can recognize epigenetic imprints at heterochromatic loci and direct heterochromatin assembly factors to newly duplicated regions marked for heterochromatin reassembly (Shibahara and Stillman 1999; Zhang et al. 2000).

We have proposed a chromatin-replication model in which modified histones and histone-associated proteins segregate semiconservatively to each DNA strand during DNA replication that could promote self-reassembly of heterochromatin on both duplicated chromosomes (Grewal and Klar 1996; Hall and Grewal 2003). Swi6 is stably associated with heterochromatin throughout the mitotic cell cycle and the entire period of meiosis (Nakayama et al. 2000). Also, Swi6 can associate with DNA polymerase $\alpha$, a component of the DNA replication machinery (Ahmed et al. 2001; Nakayama et al. 2001a), and mouse HP1 has been shown to interact with CAF-1 (Murzina et al. 1999). These findings suggest that components of heterochromatin such as Swi6/HP1 can collaborate with the DNA replication apparatus to mediate heterochromatin reassembly during DNA replication. The simplest scenario of the chromatin-assembly model entails methylated histone $\mathrm{H} 3-\mathrm{K} 9$ being stochastically redistributed to each of the two DNA strands as the replication fork passes through a heterochromatic site. Next, Swi6 can bind to the "diluted" methylated histone H3-K9 on the newly duplicated DNA molecules and helps to recruit HDACs and HMT proteins such as Clr4 to fully restore the epigenetic marks and reestablish heterochromatin to the original locus on both duplicated chromosomes. Alternatively, Swi6 and methylated histone H3-K9 are randomly transferred to the newly duplicated DNA molecules together as a unit. Interestingly, a 
number of studies have used fluorescence recovery after photobleaching (FRAP) analysis to closely monitor the kinetics of Swi6 and HP1 association with heterochromatin in real time, and all have found that the turnover rates for Swi6 and HP1 proteins are quite rapid, suggesting that the binding of Swi6/HP1 proteins to methylated H3-K9 is a dynamic event and that under conditions that could temporarily disrupt heterochromatin Swi6/HP1 can rapidly reassociate with $\mathrm{H} 3-\mathrm{K} 9$ methyl marks to reestablish heterochromatin (Cheutin et al. 2003, 2004; Festenstein et al. 2003).

In fission yeast, RNAi mutants are unable to retain heterochromatin at the centromeres, which implies the requirement for RNAi in stably maintaining heterochromatin. Indeed, our recent data suggest that stable association of RITS with chromatin is critical for the generation of RITS-associated siRNAs and the maintenance of heterochromatin at centromeres (K. Noma and S. Grewal, unpubl.). Therefore, even though heterochromatin once established is relatively stable, it tends to attenuate over time because of macrocellular processes such as DNA replication and chromosome condensation that could disrupt long-lived chromosomal structures such as heterochromatin. Consequently, there would be a continual need for heterochromatin nucleation factors, such as the RNAi machinery and/or Atf1/Pcr1, to recruit heterochromatin assembly factors, such as Clr4 and Swi6, to maintain heterochromatin so as to faithfully preserve epigenetic imprints across multiple cell divisions.

\section{BOUNDARY DETERMINANTS OF HETEROCHROMATIN}

The existence of constitutive heterochromatin domains in fission yeast and higher eukaryotes suggests the need for robust cellular mechanisms that can demarcate clear boundaries partitioning heterochromatic regions from eu- chromatic regions. In addition, chromatin domains have to be organized in a way that gives deference to a chromosome's structural constraints imposed by diverse cellular processes impinging upon the entire chromosome. For instance, chromatin surrounding the central core (cnt) and inner repeats $(\mathrm{imr})$ of $S$. pombe centromeres differ in structure and organization from the outer (otr) repeats regions (Partridge et al. 2000). These two chromatin domains are epigenetically silenced and are enriched with Mis6 and CENP-A centromeric proteins, which are essential for proper biorientation of sister centromeres during mitotic metaphase (Saitoh et al. 1997; Partridge et al. 2000; Takahashi et al. 2000). Surprisingly, Swi6 is virtually excluded from the cht and most of the imr regions and instead is confined to the outer repeats (otr) regions (Partridge et al. 2000). Evidently, a cluster of tRNA genes situated at the exterior region of imr domains act as barriers separating one silenced domain from another silenced domain with disparate functions (Partridge et al. 2000). Interestingly, tRNA genes that act as boundary elements as well have also been demonstrated in S. cerevisiae (Donze et al. 1999).

The embedded heterochromatic $K$-region of the mat locus within a larger euchromatic domain also implies the existence of boundary elements. Whereas marker genes placed within the mat $2 / 3$ interval or $K$-region are subject to epigenetic silencing, markers genes inserted outside the $K$-region are not repressed, suggesting elements present on both sides of the $K$-region could act to insulate the heterochromatic mat $2 / 3$ interval from the surrounding euchromatin. In fact, two $2-\mathrm{kb}$ identical inverted repeats termed IR-L and IR-R flank the left and right sides of the $K$-region, respectively. While $\mathrm{H} 3-\mathrm{K} 9$ methylation is associated with transcriptional silenced domains, H3-K4 methylation correlates with active transcribed regions (Strahl et al. 1999; Noma et al. 2001). Ken-ichi Noma in our lab performed a high-resolution mapping of H3-K9
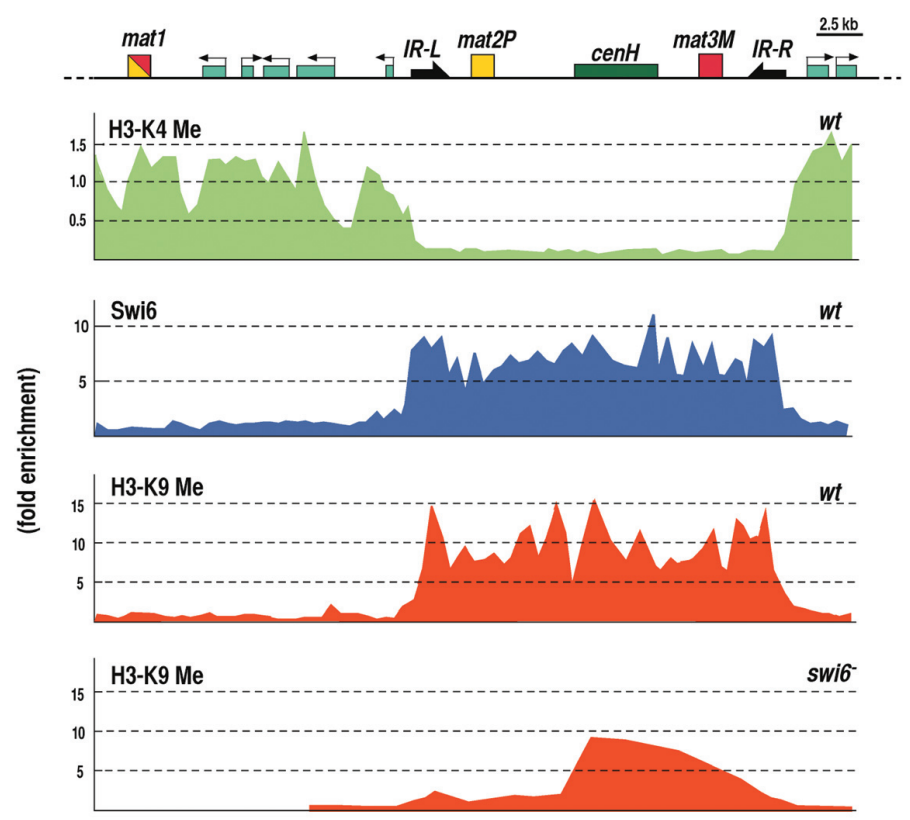

Figure 4. Distinct and mutually exclusive histone $\mathrm{H} 3-\mathrm{K} 4$ and $\mathrm{H} 3-\mathrm{K} 9$ methylation patterns respectively delimit euchromatic and heterochromatic domains at the mat locus. Distribution of H3-K4 and H3-K4 methylation patterns and Swi6 levels at the matingtype region shown in alignment with a physical map of the mat locus (top). IR-L and IR-R flanking the left and right side of the mat $2 / 3$ interval, respectively, act as boundary elements partitioning heterochromatin spanning the mat $2 / 3$ interval from surrounding euchromatin regions. Loss of Swi6 prevents the spreading of H3-K9 methylation beyond the left side of cenH (bottom). 
and H3-K4 methylation across the entire $47 \mathrm{~kb}$ mat locus (Noma et al. 2001). Consistent with evidence of the $K$-region being silenced, there was considerable enrichment of H3-K9 methylation throughout the mat2/3 interval while H3-K4 methylation was virtually absent within this region (Fig. 4). Noma et al. also mapped the level of Swi6 proteins across the mat locus and found its localization pattern identically paralleled $\mathrm{H} 3-\mathrm{K} 9$ methylation pattern throughout the mating-type region, confirming the idea that H3-K9-methyl marks act as binding sites for Swi6 proteins (Noma et al. 2001). Unexpectedly, there was a sudden sharp concomitant decline of H3-K9 methylation and Swi6 levels on both sides of the $K$-region where the two IR elements reside (Noma et al. 2001). Outside of the $K$-region, there was a gradual increase in H3-K4 methylation on both sides where a number of transcriptionally active loci lie. These results indicate that methylation of $\mathrm{H} 3-\mathrm{K} 4$ or H3-K9 is associated in a mutually exclusive manner with euchromatin and heterochromatin, respectively (Noma et al. 2001). Intriguingly, ChIP mapping across the 53-kb chicken $\beta$-globin locus also found similar patterns of $\mathrm{H} 3-\mathrm{K} 4$ methylation and $\mathrm{H} 3$ acetylation that are negatively correlated with the pattern of H3-K9 methylation (Litt et al. 2001).

The high level of methylated H3-K9 seen at the two IR elements on the side of the $K$-region while its level precipitously dropped at the immediate outside region of the mat 2/3 interval suggest IR-L and IR-R may act as boundaries for the heterochromatic mat $2 / 3$ interval. Indeed, removal of either IR element allows heterochromatin to spread outside of the mat $2 / 3$ interval and weakens heterochromatin at the $K$-region (Noma et al. 2001; Thon et al. 2002).

\section{CONCLUDING REMARKS}

While it has become increasingly clear that cells utilize heterochromatin to impose transcriptional silencing constitutively to a particular genomic region, the larger roles that heterochromatin plays in other cellular processessuch as chromosome segregation, suppression of recombination, and maintenance of genomic integrity - have only begun to be appreciated. For instance, the condensed structures of heterochromatin in $S$. pombe have been shown to be important for proper functioning of the centromeres and telomeres. Heterochromatin loss at the centromeres affects proper orientation and assembly of the kinetochore (Pidoux and Allshire 2000; Sullivan et al. 2001). In addition, components of heterochromatin such as Swi6 have been reported to recruit cohesin proteins to the centromeres as well as other heterochromatic regions along the chromosome arms, including the mat2/3 interval (Hall and Grewal 2003). In RNAi mutants in which heterochromatin is absent at the centromeres, centromeric cohesion was compromised, accompanied by severe mitotic and meotic segregation defects (Hall et al. 2003). Last, at the telomeres, telomere-specific silencing factors, such as Taz1 and Rap1, and RNAi components contribute to heterochromatin formation. The presence of heterochromatin at the telomeres is important for the maintenance of proper telomere length and the clustering of telomeres at the spindle pole body during meiosis (Cooper et al. 1998; Nimmo et al. 1998; Hall et al. 2003).

The many commonalities shared between heterochromatic regions of the $S$. pombe genome and genomes of other higher eukaryotes - from homologous molecular components of heterochromatin to similar processes of heterochromatin assembly and, even still, to similar higher-order chromatin organization of the genome, such as the structures of centromeres and telomeres - suggest that insights gained from the studies of heterochromatin in $S$. pombe will be equally applicable to the studies of heterochromatin in organisms with larger and more complex genomes. Particularly, in view of the fact that a relatively small portion of mammalian genomes consists of euchromatin, with euchromatic "islands" often scattered within larger heterochromatic domains, the role of heterochromatin in these systems will be vital for the normal functioning of the cell and the biology of the organism as a whole.

\section{ACKNOWLEDGMENTS}

We thank members of the Grewal laboratory for helpful discussions and Ken-ichi Noma for critical reading of the manuscript and help with figures. Research in our laboratory is supported by the National Cancer Institute.

\section{REFERENCES}

Ahmed S., Saini S., Arora S., and Singh J. 2001. Chromodomain protein Swi6-mediated role of DNA polymerase alpha in establishment of silencing in fission yeast. J. Biol. Chem. 276: 47814.

Allshire R.C., Javerzat J.P., Redhead N.J., and Cranston G. 1994. Position effect variegation at fission yeast centromeres. Cell 76: 157.

Ayoub N., Goldshmidt I., Lyakhovetsky R., and Cohen A. 2000. A fission yeast repression element cooperates with centromere-like sequences and defines a mat silent domain boundary. Genetics 156: 983.

Bannister A.J., Zegerman P., Partridge J.F., Miska E.A., Thomas J.O., Allshire R.C., and Kouzarides T. 2001. Selective recognition of methylated lysine 9 on histone H3 by the HP1 chromo domain. Nature 410: 120.

Bernstein E., Caudy A.A., Hammond S.M., and Hannon G.J. 2001. Role for a bidentate ribonuclease in the initiation step of RNA interference. Nature 409: 363.

Bjerling P., Silverstein R.A., Thon G., Caudy A., Grewal S.I.S., and Ekwall K. 2002. Functional divergence between histone deacetylases in fission yeast by distinct cellular localization and in vivo specificity. Mol. Cell. Biol. 22: 2170.

Brasher S.V., Smith B.O., Fogh R.H., Nietlispach D., Thiru A., Nielsen P.R., Broadhurst R.W., Ball L.J., Murzina N.V., and Laue E.D. 2000. The structure of mouse HP1 suggests a unique mode of single peptide recognition by the shadow chromo domain dimer. EMBO J. 19: 1587.

Cheutin T., Gorski S.A., May K.M., Singh P.B., and Misteli T. 2004. In vivo dynamics of Swi6 in yeast: Evidence for a stochastic model of heterochromatin. Mol. Cell. Biol. 24: 3157.

Cheutin T., McNairn A.J., Jenuwein T., Gilbert D.M., Singh P.B., and Misteli T. 2003. Maintenance of stable heterochromatin domains by dynamic HP1 binding. Science 299: 721 .

Cooper J.P., Watanabe Y., and Nurse P. 1998. Fission yeast Taz1 protein is required for meiotic telomere clustering and recombination. Nature 392: 828 . 
Cowieson N.P., Partridge J.F., Allshire R.C., and McLaughlin P.J. 2000. Dimerisation of a chromo shadow domain and distinctions from the chromodomain as revealed by structural analysis. Curr. Biol. 10: 517.

Donze D., Adams C.R., Rine J., and Kamakaka R.T. 1999. The boundaries of the silenced HMR domain in Saccharomyces cerevisiae. Genes Dev. 13: 698.

Egel R., Beach D.H., and Klar A.J. 1984. Genes required for initiation and resolution steps of mating-type switching in fission yeast. Proc. Natl. Acad. Sci. 81: 3481.

Festenstein R., Pagakis S.N., Hiragami K., Lyon D., Verreault A., Sekkali B., and Kioussis D. 2003. Modulation of heterochromatin protein 1 dynamics in primary mammalian cells. Science 299: 719 .

Grewal S.I.S. 2000. Transcriptional silencing in fission yeast. $J$. Cell. Physiol. 184: 311.

Grewal S.I. and Elgin S.C. 2002. Heterochromatin: New possibilities for the inheritance of structure. Curr. Opin. Genet. Dev. 12: 178 .

Grewal S.I.S. and Klar A.J. 1996. Chromosomal inheritance of epigenetic states in fission yeast during mitosis and meiosis. Cell 86: 95.

1997. A recombinationally repressed region between mat 2 and mat 3 loci shares homology to centromeric repeats and regulates directionality of mating-type switching in fission yeast. Genetics 146: 1221.

Grewal S.I.S., Bonaduce M.J., and Klar A.J. 1998. Histone deacetylase homologs regulate epigenetic inheritance of transcriptional silencing and chromosome segregation in fission yeast. Genetics 150: 563.

Hall I.M. and Grewal S.I.S. 2003. Structure and function of heterochromatin: Implications for epigenetic gene silencing and genome organization. In RNAi: A guide to gene silencing (ed. G. Hannon), p. 205. Cold Spring Harbor Laboratory Press, Cold Spring Harbor, New York.

Hall I.M., Noma K., and Grewal S.I.S. 2003. RNA interference machinery regulates chromosome dynamics during mitosis and meiosis in fission yeast. Proc. Natl. Acad. Sci. 100: 193.

Hall I.M., Shankaranarayana G.D., Noma K., Ayoub N., Cohen A., and Grewal S.I.S. 2002. Establishment and maintenance of a heterochromatin domain. Science 297: 2232.

Hammond S.M., Boettcher S., Caudy A.A., Kobayashi R., and Hannon G.J. 2001. Argonaute2, a link between genetic and biochemical analyses of RNAi. Science 293: 1146.

Hsieh J. and Fire A. 2000. Recognition and silencing of repeated DNA. Annu. Rev. Genet. 34: 187.

Jacobs S.A. and Khorasanizadeh S. 2002. Structure of HP1 chromodomain bound to a lysine 9-methylated histone H3 tail. Science 295: 2080.

Jenuwein T. and Allis C.D. 2001. Translating the histone code. Science 293: 1074.

Jia S., Noma K., and Grewal S.I.S. 2004. RNAi-independent heterochromatin nucleation by the stress-activated ATF/CREB family proteins. Science 304: 1971.

Kurdistani S.K. and Grunstein M. 2003. Histone acetylation and deacetylation in yeast. Nat. Rev. Mol. Cell Biol. 4: 276.

Lachner M., O'Carroll D., Rea S., Mechtler K., and Jenuwein T. 2001. Methylation of histone H3 lysine 9 creates a binding site for HP1 proteins. Nature 410: 116.

Litt M.D., Simpson M., Gaszner M., Allis C.D., and Felsenfeld G. 2001. Correlation between histone lysine methylation and developmental changes at the chicken beta-globin locus. Science 293: 2453.

Lorentz A., Ostermann K., Fleck O., and Schmidt H. 1994. Switching gene swi6, involved in repression of silent matingtype loci in fission yeast, encodes a homologue of chromatinassociated proteins from Drosophila and mammals. Gene 143: 139

Murchison E.P. and Hannon G.J. 2004. miRNAs on the move: miRNA biogenesis and the RNAi machinery. Curr. Opin. Cell Biol. 16: 223.

Murzina N., Verreault A., Laue E., and Stillman B. 1999. Heterochromatin dynamics in mouse cells: Interaction between chromatin assembly factor 1 and HP1 proteins. Mol. Cell 4:
529.

Nakayama J., Klar A.J., and Grewal S.I.S. 2000. A chromodomain protein, Swi6, performs imprinting functions in fission yeast during mitosis and meiosis. Cell 101: 307.

Nakayama J., Allshire R.C., Klar A.J., and Grewal S.I.S. 2001a. A role for DNA polymerase alpha in epigenetic control of transcriptional silencing in fission yeast. EMBO J. 20: 2857.

Nakayama J., Rice J.C., Strahl B.D., Allis C.D., and Grewal S.I.S. 2001b. Role of histone H3 lysine 9 methylation in epigenetic control of heterochromatin assembly. Science 292: 110.

Nakayama J., Xiao G., Noma K., Malikzay A., Bjerling P., Ekwall K., Kobayashi R., and Grewal S.I.S. 2003. Alp13, an MRG family protein, is a component of fission yeast Clr6 histone deacetylase required for genomic integrity. $E M B O J .22$ : 2776.

Nielsen P.R., Nietlispach D., Mott H.R., Callaghan J., Bannister A., Kouzarides T., Murzin A.G., Murzina N.V., and Laue E.D. 2002. Structure of the HP1 chromodomain bound to histone $\mathrm{H} 3$ methylated at lysine 9. Nature 416: 103.

Nimmo E.R., Pidoux A.L., Perry P.E., and Allshire R.C. 1998. Defective meiosis in telomere-silencing mutants of Schizosaccharomyces pombe (comments). Nature 392: 825.

Noma K., Allis C.D., and Grewal S.I.S. 2001. Transitions in distinct histone $\mathrm{H} 3$ methylation patterns at the heterochromatin domain boundaries. Science 293: 1150.

Partridge J.F., Borgstrom B., and Allshire R.C. 2000. Distinct protein interaction domains and protein spreading in a complex centromere. Genes Dev. 14: 783.

Partridge J.F., Scott K.S., Bannister A.J., Kouzarides T., and Allshire R.C. 2002. cis-acting DNA from fission yeast centromeres mediates histone $\mathrm{H} 3$ methylation and recruitment of silencing factors and cohesin to an ectopic site. Curr. Biol. 12: 1652.

Pidoux A.L. and Allshire R.C. 2000. Centromeres: Getting a grip of chromosomes. Curr. Opin. Cell Biol. 12: 308.

Rea S., Eisenhaber F., O'Carroll D., Strahl B.D., Sun Z.W., Schmid M., Opravil S., Mechtler K., Ponting C.P., Allis C.D., and Jenuwein T. 2000. Regulation of chromatin structure by site-specific histone H3 methyltransferases. Nature 406: 593.

Reinhart B.J. and Bartel D.P. 2002. Small RNAs correspond to centromere heterochromatic repeats. Science 297: 1831.

Saitoh S., Takahashi K., and Yanagida M. 1997. Mis6, a fission yeast inner centromere protein, acts during G1/S and forms specialized chromatin required for equal segregation. Cell 90: 131.

Schubeler D., MacAlpine D.M., Scalzo D., Wirbelauer C., Kooperberg C., van Leeuwen F., Gottschling D.E., O’Neill L.P., Turner B.M., Delrow J., Bell S.P., and Groudine M. 2004. The histone modification pattern of active genes revealed through genome-wide chromatin analysis of a higher eukaryote. Genes Dev. 18: 1263.

Selker E.U. 1999. Gene silencing repeats that count. Cell 97: 157.

Shankaranarayana G.D., Motamedi M.R., Moazed D., and Grewal S.I.S. 2003. Sir2 regulates histone H3 lysine 9 methylation and heterochromatin assembly in fission yeast. Curr. Biol. 13: 1240.

Shibahara K. and Stillman B. 1999. Replication-dependent marking of DNA by PCNA facilitates CAF-1-coupled inheritance of chromatin. Cell 96: 575.

Strahl B.D. and Allis C.D. 2000. The language of covalent histone modifications. Nature 403: 41.

Strahl B.D., Ohba R., Cook R.G., and Allis C.D. 1999. Methylation of histone $\mathrm{H} 3$ at lysine 4 is highly conserved and correlates with transcriptionally active nuclei in Tetrahymena. Proc. Natl. Acad. Sci. 96: 14967.

Sullivan B.A., Blower M.D., and Karpen G.H. 2001. Determining centromere identity: Cyclical stories and forking paths. Nat. Rev. Genet. 2: 584.

Takahashi K., Chen E.S., and Yanagida M. 2000. Requirement of Mis6 centromere connector for localizing a CENP-A-like protein in fission yeast. Science 288: 2215.

Takahashi K., Murakami S., Chikashige Y., Funabiki H., Niwa O., and Yanagida M. 1992. A low copy number central se- 
quence with strict symmetry and unusual chromatin structure in fission yeast centromere. Mol. Biol. Cell 3: 819.

Thon G. and Verhein-Hansen J. 2000. Four chromo-domain proteins of Schizosaccharomyces pombe differentially repress transcription at various chromosomal locations. Genetics 155: 551.

Thon G., Bjerling P., Bunner C.M., and Verhein-Hansen J. 2002. Expression-state boundaries in the mating-type region of fission yeast. Genetics 161: 611 .

Tuzon C.T., Borgstrom B., Weilguny D., Egel R., Cooper J.P., and Nielsen O. 2004. The fission yeast heterochromatin protein Rik1 is required for telomere clustering during meiosis. $J$. Cell Biol. 165: 759.
Verdel A., Jia S., Gerber S., Sugiyama T., Gygi S., Grewal S.I.S., and Moazed D. 2004. RNAi-mediated targeting of heterochromatin by the RITS complex. Science 303: 672 .

Volpe T.A., Kidner C., Hall I.M., Teng G., Grewal S.I.S., and Martienssen R.A. 2002. Regulation of heterochromatic silencing and histone H3 lysine-9 methylation by RNAi. Science 297: 1833.

Wang G., Ma A., Chow C.M., Horsley D., Brown N.R., Cowell I.G., and Singh P.B. 2000. Conservation of heterochromatin protein 1 function. Mol. Cell. Biol. 20: 6970.

Zhang Z., Shibahara K., and Stillman B. 2000. PCNA connects DNA replication to epigenetic inheritance in yeast. Nature 408: 221. 


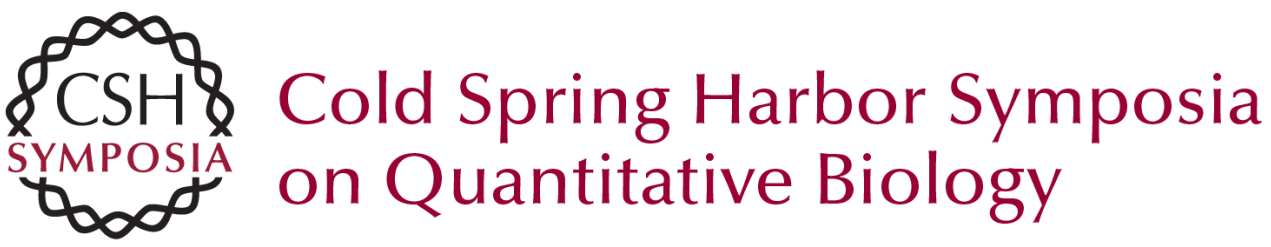

\section{RNA Interference and Epigenetic Control of Heterochromatin Assembly in Fission Yeast}

H. CAM and S.I.S. GREWAL

Cold Spring Harb Symp Quant Biol 2004 69: 419-428

Access the most recent version at doi:10.1101/sqb.2004.69.419

References This article cites 61 articles, 34 of which can be accessed free at: http://symposium.cshlp.org/content/69/419.full.html\#ref-list-1

License

Email Alerting Receive free email alerts when new articles cite this article - sign up in Service the box at the top right corner of the article or click here. 